\title{
Correction of Fe-55 Activity Measurements Due to Interference of Other Low Energy Photon Emitters
}

\author{
JAMES E. MARTIN and MICHAEL D. K. LIAW \\ School of Public Health, University of Michigan, Ann Arbor, MI 48109-2029, U.S.A.
}

(Received 2 March 1993; in revised form 6 April 1993)

\begin{abstract}
Measurement of ${ }^{55} \mathrm{Fe}$ in reactor waste samples by chemical separation of $\mathrm{Fe}$ is subject to interference by ${ }^{58} \mathrm{Co}$ and ${ }^{54} \mathrm{Mn}$ because these nuclides are produced in copious quantities in reactors, they emit $\mathrm{K} \mathrm{X}$-rays of essentially the same energy as ${ }^{55} \mathrm{Fe}$-rays, and chemical separations of $\mathrm{Fe}$ are not pure enough to assure their absence. Fortunately ${ }^{58} \mathrm{Co}$ and ${ }^{54} \mathrm{Mn}$ also emit high energy gamma rays that can be used to detect and quantitate their presence in separated samples. $\mathrm{x}$-Ray fluorescence yields of ${ }^{58} \mathrm{Co},{ }^{54} \mathrm{Mn}$ and ${ }^{55} \mathrm{Fe}$ can be used to correct count rates and provide corrected measurements of ${ }^{55} \mathrm{Fe}$. This technique has been used successfully to correct measurements of ${ }^{55} \mathrm{Fe}$ in reactor wastes, especially spent ion-exchange resins though most corrections have been only a few percent.
\end{abstract}

\section{Introduction}

Iron-55 is a common constituent in radioactive waste samples from nuclear power stations, and because of its concentrations in waste streams, its quantity must be established to comply with shipment regulations (NRC 82). It is produced by neutron activation of stable ${ }^{54} \mathrm{Fe}$ which exists in many reactor components. ${ }^{55} \mathrm{Fe}$ has a half-life of 2.73 years and decays solely by electron capture followed by emission of characteristic $\mathrm{Mn} \mathrm{x}$-rays of $5.89-6.49 \mathrm{keV}$ (ICRP, 1983). Measurement of ${ }^{55} \mathrm{Fe}$ is based on chemical separation of Fe in the sample (Martin et al., 1988) followed by detection of the $\mathrm{K}$ x-rays. Unfortunately, such chemical separations are not pure, and cobalt and nickel can contaminate the procedure. Since ${ }^{58} \mathrm{Co}$ and ${ }^{54} \mathrm{Mn}$ also emit $\mathrm{K} \mathrm{x}$-rays of similar energy, their presence can distort the ${ }^{55} \mathrm{Fe}$ measurement unless their contribution to detector response is subtracted. The purpose of this paper is to describe an approach for correcting ${ }^{55} \mathrm{Fe}$ measurements for potential contamination by ${ }^{58} \mathrm{Co}$ and ${ }^{54} \mathrm{Mn}$, both of which can exist in large concentrations in waste samples.

Cobalt-58 and ${ }^{54} \mathrm{Mn}$ are also produced in reactors by $(\mathrm{n}, \mathrm{p})$ reactions in ${ }^{58} \mathrm{Ni}$ and ${ }^{54} \mathrm{Fe}$, respectively, which are common constituents of stainless steel used in reactor components. Cobalt- 58 decays by electron capture and positron emission, followed by a gamma ray of $810.8 \mathrm{keV}$ and low energy $\mathrm{K} x$-rays between 6.4 and $7.06 \mathrm{keV}$ (Brown and Firestone, 1986); ${ }^{54} \mathrm{Mn}$ also decays by electron capture followed by a gamma ray of $834.8 \mathrm{keV}$ and $\mathrm{K}$ shell $\mathrm{x}$-rays of $5.41-5.95 \mathrm{keV}$ (Brown and Firestone, 1986). The energies of these photon transitions are listed in Table 1. These lowenergy photons from ${ }^{58} \mathrm{Co}$ and ${ }^{54} \mathrm{Mn}$ are virtually indistinguishable from those of ${ }^{55} \mathrm{Fe}$ when counted by a germanium detector at high gain. Figure 1 shows spectra of the $\mathrm{x}$-rays for each; thus, if either happens to be present in the separated sample, they will interfere with the measurement. However, one can use the gamma ray emissions from ${ }^{58} \mathrm{Co}$ and ${ }^{54} \mathrm{Mn}$ to quantitate their amounts in waste sample separations for ${ }^{55} \mathrm{Fe}$ such that their $\mathrm{x}$-ray contributions to the ${ }^{55} \mathrm{Fe}$ measurement can be subtracted.

\section{Method}

Iron-55 separation was performed for waste samples from two local utilities by the protocol developed by Martin et al. (1988). Separation of ${ }^{55} \mathrm{Fe}$ was achieved by solvent extraction using triisocytlamine in xylene. Low-energy photon counting of the approx. $6 \mathrm{keV}{ }^{55} \mathrm{Mn} x$-rays was done by a bare intrinsic germanium detector with a high gain setting and $3-\mu$ s shaping time to identify and quantitate the ${ }^{55} \mathrm{Fe}$ peak. Contaminants of ${ }^{58} \mathrm{Co}$ and ${ }^{54} \mathrm{Mn}$ which may be carried over in the chemical separation can be detected by measuring the gamma-ray emissions.

After the initial radiochemical separation, ${ }^{55} \mathrm{Fe}$ was precipitated onto a Gelman A-E filter and allowed to dry before counting. Samples were placed in plastic bags to prevent contamination of the detector. The samples (filter paper and plastic bag) were counted for $10 \mathrm{~min}$ on the intrinsic germanium detector under routine settings to quantitate ${ }^{58} \mathrm{Co}$ (photopeak $=$ 


\begin{tabular}{lcccccc}
\multicolumn{4}{c}{ Table 1. Photon emission by ${ }^{58} \mathrm{Co},{ }^{54} \mathrm{Mn}$ and ${ }^{55} \mathrm{Fe}$} \\
\hline \multicolumn{3}{c}{${ }^{58} \mathrm{Co}$} \\
Photon & $E(\mathrm{kev})$ & $Y(\%)$ & $E(\mathrm{kev})$ & $Y(\%)$ & $E(\mathrm{kev})$ & $Y(\%)$ \\
\hline$K_{\mathrm{a}}$ x-ray & 6.40 & 23.00 & 5.41 & 22.00 & 5.90 & 24.40 \\
$K_{\beta}$ x-1ay & 7.06 & 2.75 & 5.95 & 2.47 & 6.49 & 2.86 \\
Gamma & 810.8 & 99.5 & 834.8 & 100 & 0 & 0 \\
\hline * $Y$, percent photon yield per nuclear transformation (Browne and Firestone. 1986).
\end{tabular}

$810.8 \mathrm{keV}$ ) and ${ }^{54} \mathrm{Mn} \quad$ (photopeak $=834.8 \mathrm{keV}$ ) using a detector efficiency file determined by a mixed gamma-ray standard traceable to the National Institute of Standards and Technology (NIST).

The disintegration rate of ${ }^{58} \mathrm{Co}$ and ${ }^{54} \mathrm{Mn}$ determined by gamma counting allows determination of the emission rate of characteristic $x$-rays from these contaminants. These emission rates are determined by multiplying the disintegration rate by the photon yields listed in Table 1 . Both $K_{\mathrm{a}}$ and $K_{p}$ x-rays are accounted for in the calculation. These emission rates are converted into detected counts by multiplying the emission rate by the photon counting efficiency at the energy of these $\mathrm{x}$-rays, which was determined by calibration of the detector in this low energy region with a standard of ${ }^{55} \mathrm{Fe}$. The overall counting efficiency of ${ }^{55} \mathrm{Fe}$ is actually a combination of efficiencies for both $K_{\mathrm{a}}$ and $K_{\beta}$ x-rays since they are of similar energy. In determining the total counting efficiency of ${ }^{55} \mathrm{Fe}$, it is also necessary to use both the $K_{\mathrm{a}}$ and $K_{p}$ $\mathrm{x}$-ray yields. A piece of filter paper with a known quantity of ${ }^{55} \mathrm{Fe}$ activity was counted by the germanium detector. The fractional yield of $\mathrm{K}$ x-rays per nuclear transformation of ${ }^{55} \mathrm{Fe}$ was $27.3 \%$ (see Table 1) and the detector efficiency for $6 \mathrm{keV}$ photons was $6.70 \%$; thus, when a standard of ${ }^{55} \mathrm{Fe}$ is counted, 0.0181 counts per nuclear transformation of ${ }^{55} \mathrm{Fe}$ are observed.

For example, 10-min counts of an iron separation of a resin sample on the germanium detector indicated $46,712 \mathrm{cpm}$ of ${ }^{55} \mathrm{Fe}, 142.2 \mathrm{cpm}$ of ${ }^{58} \mathrm{Co}$, and $267.5 \mathrm{cpm}$ of ${ }^{54} \mathrm{Mn}$. Detector efficiency data $(0.75 \%$ for ${ }^{58} \mathrm{Co} ; 0.73 \%$ for ${ }^{54} \mathrm{Mn}$ ) and the data in Table 1 were used to correct the count rate due to ${ }^{55} \mathrm{Fe}$ to $45,784 \mathrm{cpm}$.

\section{Summary}

Measurement of ${ }^{55} \mathrm{Fe}$ in reactor waste samples by chemical separation of $\mathrm{Fe}$ is subject to interference by ${ }^{58} \mathrm{Co}$ and ${ }^{54} \mathrm{Mn}$ because these nuclides are produced in copious quantities in reactors, they emit $\mathrm{K} \mathrm{X}$-rays of essentially the same energy as ${ }^{55} \mathrm{Fe}$ $\mathrm{x}$-rays which interfere with the counting technique, and chemical separations of Fe are not pure enough to assure their absence. Fortunately ${ }^{58} \mathrm{Co}$ and ${ }^{54} \mathrm{Mn}$ also emit high energy gamma rays that can

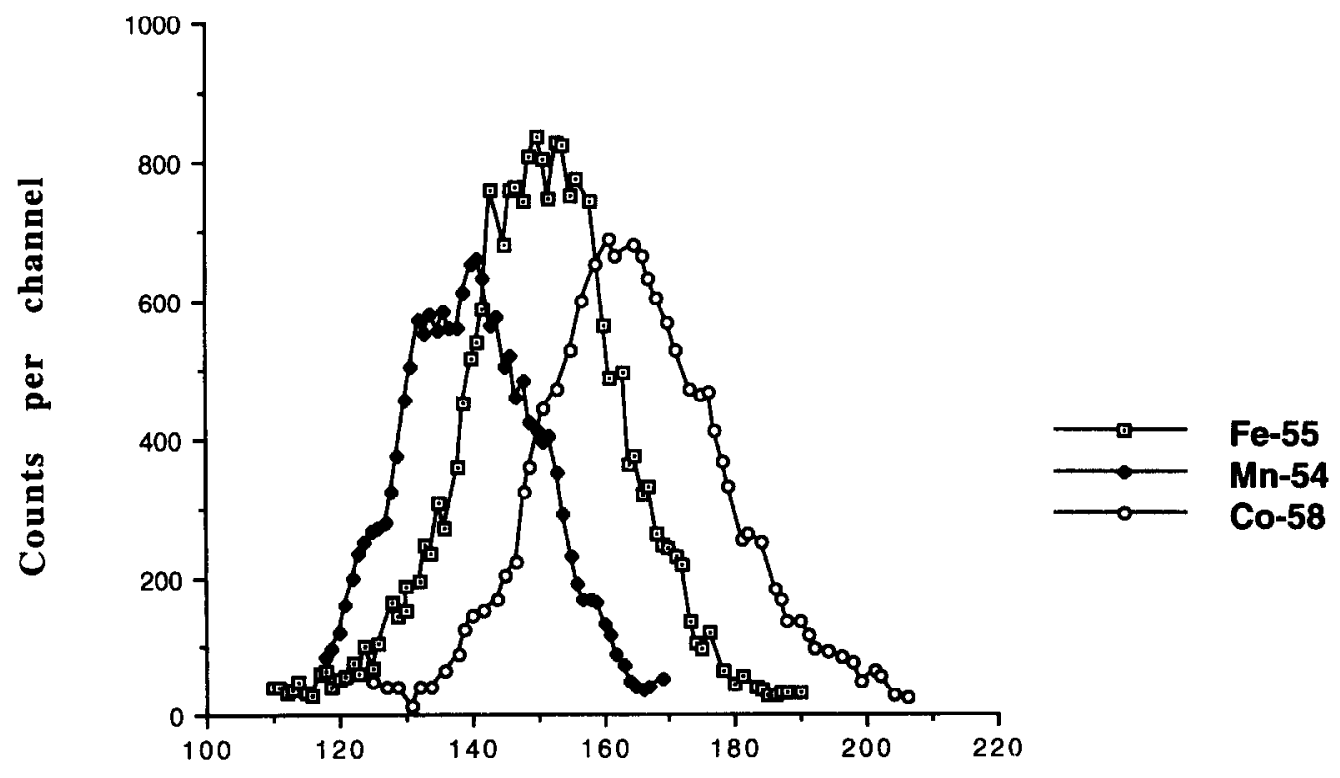

\section{Channel numbers}

Fig. 1. Normalized photon spectra of ${ }^{55} \mathrm{Fe},{ }^{54} \mathrm{Mn}$ and ${ }^{58} \mathrm{Co}$ obtained with a germanium detector and high gain. 
be used to detect and quantitate their presence in separated samples. $x$-Ray fluorescence yields of ${ }^{58} \mathrm{Co},{ }^{54} \mathrm{Mn}$ and ${ }^{55} \mathrm{Fe}$ can be used to correct count rates and provide corrected measurements of ${ }^{55} \mathrm{Fe}$. This technique has been used successfully for ${ }^{55} \mathrm{Fe}$ in reactor wastes, especially spent ion-exchange resins, though most corrections have been only a few percent.

\section{References}

Browne E. and Firestone R. B. (1986) Table of Radioactive Isotopes (Ed. Shirley V. S.). Wiley, New York.

International Commission on Radiological Protection (ICRP) (1983) Publication 38: Radionuclide transformations: Energy and Intensity of Emission.

Martin J. E., Hylko J. M. and Jones J. D. (1988) Measurement of ${ }^{55} \mathrm{Fe}$ in reactor samples by an intrinsic germanium detector. Appl. Radiat. Isot. 38, 28. 